\title{
Mappings of finite distortion: discreteness and openness for quasi-light mappings
}

\section{Applications à distortion finie: les applications "quasi-light" sout discrètes et ouvertes}

\author{
Stanislav Hencl *, Pekka Koskela \\ Department of Mathematics and Statistics, University of Jyväskylä, PO Box 35 (MaD), FIN-40014, Jyväskylä, Finland
}

Received 2 February 2004; accepted 6 July 2004

Available online 7 April 2005

\begin{abstract}
Let $f \in W^{1, n}\left(\Omega, \mathbb{R}^{n}\right)$ be a continuous mapping so that the components of the preimage of each $y \in \mathbb{R}^{n}$ are compact. We show that $f$ is open and discrete if $|D f(x)|^{n} \leqslant K(x) J_{f}(x)$ a.e. where $K(x) \geqslant 1$ and $K^{n-1} / \Phi(\log (e+K)) \in L^{1}(\Omega)$ for a function $\Phi$ that satisfies $\int_{1}^{\infty} 1 / \Phi(t) \mathrm{d} t=\infty$ and some technical conditions. This divergence condition on $\Phi$ is shown to be sharp.

(C) 2005 L'Association Publications de l'Institut Henri Poincaré. Published by Elsevier B.V. All rights reserved
\end{abstract}

\section{Résumé}

Soit $f \in W^{1, n}\left(\Omega, \mathbb{R}^{n}\right)$ une application continue telle que les composantes connexes de la préimage de tat point $y \in \mathbb{R}^{n}$ est compacte. On démontre que $f$ est ouverte et discrète si $|D f(x)|^{n} \leqslant K(x) J_{f}(x)$ p.p. oi $K(x) \geqslant 1$ et $K^{n-1} / \Phi(\log (e+K)) \in$ $L^{1}(\Omega)$ pour une fonction $\Phi$ telle que $\int_{1}^{\infty} 1 / \Phi(t) \mathrm{d} t=\infty$ et vérifiant des conditions techniques. La condition de divergence de l'intégrale est optimale.

(C) 2005 L'Association Publications de l'Institut Henri Poincaré. Published by Elsevier B.V. All rights reserved

MSC: 30C65; 26B10; 73C50

Keywords: Mapping of finite distortion; Discreteness; Openness

\footnotetext{
* Corresponding author.

E-mail addresses: henc1@maths.jyu.fi (S. Hencl), pkoskela@maths.jyu.fi (P. Koskela).
} 


\section{Introduction}

Let $\Omega$ be a connected, open subset of $\mathbb{R}^{n}$ with $n \geqslant 2$. In this paper we consider continuous mappings $f \in W_{\text {loc }}^{1, p}\left(\Omega, \mathbb{R}^{n}\right), p \geqslant 1$. We suppose that there is a measurable function $K: \Omega \rightarrow[1, \infty)$ so that the distortion inequality

$$
|D f(x)|^{n} \leqslant K(x) J_{f}(x)
$$

holds almost everywhere in $\Omega$, where $D f(x)$ is the differential matrix of $f$ at $x,|D f(x)|$ is the operator norm of this matrix, and $J_{f}(x)$ is the determinant of $D f(x)$. We say that $f$ is of finite distortion $K$ if furthermore $J_{f}$ is locally integrable.

If above $K$ is bounded, then necessarily $f \in W_{\text {loc }}^{1, n}\left(\Omega, \mathbb{R}^{n}\right)$, and we recover the class of mappings of bounded distortion, also called quasiregular mappings (cf. $[22,23,9,26]$ ). One of the fundamental properties of mappings of bounded distortion is the remarkable result by Reshetnyak [21] that such a mapping is either constant or both discrete and open. This means that the preimage of each point is a discrete set of points and that $f$ maps open sets to open sets.

A principal goal in the theory of mappings of finite distortion has been to try and obtain analogs of Reshetnyak's result. In [10] Iwaniec and Šverák proved in dimension two, using the Beltrami equation, that each non-constant mapping $f \in W^{1,2}\left(\Omega, \mathbb{R}^{2}\right)$ of finite distortion $K \in L^{1}(\Omega)$ is both open and discrete. Subsequently, Heinonen and Koskela [6] proved in higher dimensions that a quasi-light mapping $f \in W_{\text {loc }}^{1, n}\left(\Omega, \mathbb{R}^{n}\right)$ of finite distortion $K \in L^{p}(\Omega), p>n-1$, is open and discrete. Here the quasi-lightness means that the components of the preimage of each $y \in \mathbb{R}^{n}$ are compact. Manfredi and Villamor [20] then showed that the quasi-lightness assumption can be disposed of. The most recent result in this direction is due to Hencl and Malý [7]. They showed that, for a quasilight mapping $f \in W_{\text {loc }}^{1, n}\left(\Omega, \mathbb{R}^{n}\right)$ of finite distortion, the integrability assumption $K \in L^{n-1}(\Omega)$ is sufficient for discreteness and openness. The general case remains open.

It is then natural to inquire if the integrability of $K$ could be further relaxed. To this end, let us first recall a construction by Ball [1]. He gives an example of a non-constant, Lipschitz continuous quasi-light mapping of finite distortion $K$, defined in a domain $\Omega$, so that $f$ maps a line segment to a point and with $K \in L^{p}(\Omega)$ for all $p<n-1$. Moreover, the preimage of every other point consists of at most a single point. Regarding the distortion function $K$, one can in fact check that

$$
\frac{K^{n-1}}{\Phi(\log (e+K))} \in L^{1}(\Omega)
$$

whenever $\int_{1}^{\infty} \frac{\mathrm{d} t}{\Phi(t)}<\infty$. Iwaniec and Martin [9] have conjectured that each non-constant mapping $f \in$ $W_{\text {loc }}^{1, n}\left(\Omega, \mathbb{R}^{n}\right)$ of finite distortion that satisfies (1.1) for some (sufficiently regular $\Phi$ ) with $\int_{1}^{\infty} \frac{\mathrm{d} t}{\Phi(t)}=\infty$ is in fact both open and discrete; in fact their conjecture is slightly stronger because it involves a different distortion function. However, this far there have been no results under assumptions weaker than $K \in L^{n-1}(\Omega)$, even for quasi-light mappings. We give the first step towards to this conjecture by establishing the following sharp result.

Suppose that we are given a function $\Phi:[1, \infty) \rightarrow(0, \infty)$ such that

(i) $\Phi$ is continuous and non-decreasing,

$$
\int_{1}^{\infty} \frac{\mathrm{d} t}{\Phi(t)}=\infty,
$$

(iii) the function $t \rightarrow t^{n-1} / \Phi(\log (e+t))$ is increasing,

(iv) for every $c_{1}>0$ there is $c_{2}>0$ such that $\Phi\left(c_{1} t\right) \leqslant c_{2} \Phi(t)$.

Notice that these conditions are satisfied for example for $\Phi_{1}(t)=1, \Phi_{2}(t)=t, \Phi_{3}(t)=t \log (e+t)$ and so on. 
Theorem 1.1. Let $\Omega \subset \mathbb{R}^{n}$ be a connected open set. Suppose that $f \in W_{\text {loc }}^{1, n}\left(\Omega, \mathbb{R}^{n}\right)$ is a quasi-light mapping of finite distortion $K$ that satisfies (1.1) with a function $\Phi$ that satisfies (1.2). Then $f$ is open and discrete.

Before discussing the proof of Theorem 1.1, let us briefly comment on the regularity assumptions on $f$. First of all, there exists a quasi-light mapping $f$ of finite distortion $K$ that fails to be discrete and open, satisfies $f \in W^{1, p}\left(\Omega, \mathbb{R}^{n}\right)$ for all $p<n$ and satisfies $K \in L^{p}(\Omega)$ for all $p<\infty$. Thus the regularity assumption $f \in W_{\text {loc }}^{1, n}\left(\Omega, \mathbb{R}^{n}\right)$ cannot be substantially relaxed. Such mappings have been constructed in $[12,14]$. We prove a stronger version of Theorem 1.1 in Section 5 that comes with an optimal regularity assumption. Secondly, we have taken continuity as a standing assumption for mappings of finite distortion. Under the regularity assumptions referred to above, continuity follows from the other assumptions, as was shown in $[4,8,14]$.

All the proofs of discreteness and openness for $n \geqslant 3$ that we are aware of rely on the following idea. One first proves that the mapping in question is sense-preserving. After that one verifies that the preimage of each $y \in \mathbb{R}^{n}$ is totally disconnected. The claim then follows by invoking the Titus-Young theorem [24]. We follow this procedure. The fact that $f$ be sense-preserving in the setting of Theorem 1.1 is already due to Reshetnyak and the more general case can be essentially found in $[12,14]$. Thus we are reduced to showing that the preimage of each $y \in \mathbb{R}^{n}$ is totally disconnected. This will be guaranteed by our following theorem.

Theorem 1.2. Let $\Omega \subset \mathbb{R}^{n}$ be a connected open set and suppose that $f \in W_{\text {loc }}^{1, s}\left(\Omega, \mathbb{R}^{n}\right), n-1<s \leqslant n$, is a mapping of finite distortion K. Furthermore, assume that $K$ satisfies (1.1) with a function $\Phi$ that satisfies (1.2) and that the multiplicity of $f$ is essentially bounded in a neighborhood of 0 . Then either $f \equiv 0$ or $H^{1}\left(f^{-1}(0)\right)=0$.

The essential boundedness of the multiplicity means that there is an integer $k$ so that the cardinality of $f^{-1}(y)$ is at most $k$ for almost all $y$ in the given neighborhood of 0 . The fact that we can bound the multiplicity under our assumptions is based on certain results in $[12,13,7]$.

We prove Theorem 1.2 by first establishing a sharp generalization of an oscillation estimate given in [7]. We believe that this oscillation estimate, given in Section 3, is of its own interest. Indeed, the original version has already found applications [18]. Our estimate is, in a sense, a substitute for the usual bounds on capacity in terms of Hausdorff measures. The usual bounds are not subtle enough for our purposes. Theorem 1.2 is then obtained by combining the oscillation estimate with a delicate integrability result on $|f|^{-1}$.

Theorem 1.2 is very sharp. The integrability assumption on $K$ cannot be relaxed because of the example due to Ball, mentioned above. Moreover, we cannot take $s=n-1$ at least when $n=2$, as is seen by considering the mapping defined by $f(x)=x / \log (e /(|x|-1))$ for $x \in B(0,2) \backslash B(0,1)$ and by $f(x)=0$ for $x \in \bar{B}(0,1)$. Indeed, $f$ is of finite distortion $K$ with $K / \Phi(\log (e+K)) \in L^{1}(B(0,2))$ for, say, $\Phi(t)=t$, and the multiplicity of $f$ is essentially bounded by one in any neighborhood of 0 .

The paper is organized as follows. In Section 2 we recall some definitions and preliminary results. Section 3 is devoted to the proofs of oscillation estimates. In Section 4, we prove Theorem 1.2. Finally, in Section 5, we discuss discreteness and openness and give the proof of Theorem 1.1.

\section{Preliminaries}

\subsection{Quasicontinuous representatives}

In anticipation of future applications of our oscillation estimates, we will formulate them without the continuity assumption. This will be done in terms of quasicontinuous representatives. Let us point out that each continuous Sobolev mapping is quasicontinuous. In this paper, the precise definition of such a representative of a Sobolev mapping is not needed, because we will only employ the following fact [7, Proposition 1]. See [19] for more on quasicontinuity. 
Proposition 2.1. Let $1 \leqslant p<\tilde{p} \leqslant n$. Let $u \in W^{1, \tilde{p}}(\Omega)$ be $1, \tilde{p}$-quasicontinuous. Then for $H^{n-p}$-a.e. point $z \in \Omega$ we have

$\limsup _{r \rightarrow 0} r^{-\beta} f_{B(z, r)}|u-u(z)| \mathrm{d} x<\infty$,

where $\beta=1-p / \tilde{p}$.

\subsection{Topological properties}

A mapping $f: \Omega \rightarrow \mathbb{R}^{n}$ is said to be discrete if the preimage of each point of $\mathbb{R}^{n}$ is locally finite in $\Omega$, and light if the preimage of each point of $\mathbb{R}^{n}$ is totally disconnected. We say that $f: \Omega \rightarrow \mathbb{R}^{n}$ is quasi-light if for each $y \in \mathbb{R}^{n}$ the components of the set $f^{-1}(y)$ are compact. We call a continuous mapping $f: \Omega \rightarrow \mathbb{R}^{n}$ sensepreserving if $\operatorname{deg}\left(f, \Omega^{\prime}, y_{0}\right)>0$ for all domains $\Omega^{\prime} \Subset \Omega$ and all $y_{0} \in f\left(\Omega^{\prime}\right) \backslash f\left(\partial \Omega^{\prime}\right)$, where $\operatorname{deg}\left(f, \Omega^{\prime}, y_{0}\right)$ is the topological degree of $f$ at $y_{0}$ with respect to $\Omega^{\prime}$. For the definition of the topological degree see e.g. [3].

\subsection{Area formula}

We denote by $|E|$ the Lebesgue measure of a set $E \subset \mathbb{R}^{n}$. We will use the well-known area formula. Let $f \in W_{\text {loc }}^{1,1}\left(\Omega ; \mathbb{R}^{n}\right)$. The multiplicity function $N(f, \Omega, y)$ of $f$ is defined as the number of preimages of $y$ under $f$ in $\Omega$. Let $\eta$ be a nonnegative Borel measurable function on $\mathbb{R}^{n}$. Without any additional assumption we have

$$
\int_{\Omega} \eta(f(x))\left|J_{f}(x)\right| \mathrm{d} x \leqslant \int_{\mathbb{R}^{n}} \eta(y) N(f, \Omega, y) \mathrm{d} y .
$$

This follows from the area formula for Lipschitz mappings, from the a.e. approximate differentiability of $f$ [2, Theorem 3.1.4], and a general property of a.e. approximately differentiable functions [2, Theorem 3.1.8], namely that $\Omega$ can be exhausted up to a set of measure zero by sets the restriction to which of $f$ is Lipschitz continuous.

\subsection{Fine properties of Hausdorff measure}

In the proof of the oscillation estimate we need the following set functions:

$$
\lambda_{\delta}^{d}: E \mapsto \inf \left\{\sum_{\alpha} a_{\alpha}\left(\operatorname{diam} E_{\alpha}\right)^{d}: a_{\alpha} \geqslant 0, \operatorname{diam} E_{\alpha} \leqslant \delta, \chi_{E} \leqslant \sum_{\alpha} a_{\alpha} \chi_{E_{\alpha}}\right\}, \quad \delta>0 .
$$

By $[2,2.10 .24]$,

$$
\lim _{\delta \rightarrow 0} \lambda_{\delta}^{d}(E)=H^{d}(E)
$$

for any set $E \subset \mathbb{R}^{n}$, where $H^{d}$ is the usual Hausdorff measure.

\section{Divergence criterion}

If we assume, for $p<n$, that a non-negative function $u \in W^{1, p}(\Omega)$ vanishes sufficiently fast on a set of positive $(n-p)$-dimensional Hausdorff measure (it suffices to assume a certain power-like decay of integral means of $u$ over $B(x, r)$ as $r \rightarrow 0)$, then we can find pairwise disjoint sets $E_{i}$ such that $\left|E_{i}\right|>0$ and

$$
\sup _{E_{i}} u^{p} \leqslant C \int_{E_{i}}|\nabla u|^{p} \mathrm{~d} x
$$


(see [7, Theorems 3 and 4] for the exact statement, and also [17]). By a small modification of the proofs of those results one can obtain $\inf _{E_{i}} u>C \sup _{E_{i}} u$, and, therefore

$$
\int_{\Omega} \frac{|\nabla u|^{p}}{u^{p}} \mathrm{~d} x=\infty .
$$

We generalize this fact in the next lemma by obtaining a sharp divergence statement (3.4).

Lemma 3.1. Let $1 \leqslant p<n, \mu>0, \beta \in(0,1)$ and $\gamma>0$. Let $\Omega \subset \mathbb{R}^{n}$ be a connected open set and $u \in W^{1, p}(\Omega)$. Suppose that $u>0$ a.e. and let

$$
Z=\left\{z \in \Omega: \limsup _{r \rightarrow 0} r^{-\beta} f_{B(z, r)} u \mathrm{~d} x<\gamma\right\} .
$$

Suppose that $H^{n-p}(Z)>\mu$. Suppose further that a function $\Phi:[1, \infty) \rightarrow(0, \infty)$ satisfies

(i) for every $c_{1}>0$ there is $c_{2}>0$ such that for every $l \in \mathbb{N} \Phi\left(c_{1}(l+1)\right) \geqslant c_{2} \Phi\left(c_{1} l\right)$,

(ii) $\int_{1}^{\infty} \frac{\mathrm{d} t}{\Phi(t)}=\infty$.

Then, for each $0<\delta<\mathrm{e}^{-e}$,

$$
\int_{0<u<\delta} \frac{|\nabla u|^{p}}{u^{p} \log 1 / u \Phi(\log \log 1 / u)} \mathrm{d} x=\infty .
$$

Proof. Our argument is an improvement on [7, Theorem 3]. We will omit the parts of the reasoning there that need not be altered. Set

$$
\tau:=2^{\beta / 4} \text {. }
$$

For $j, m \in \mathbb{Z}$ we denote

$$
\begin{aligned}
& Z_{m}=\left\{z \in Z: B\left(z, 2^{-m}\right) \subset \Omega, 2^{m^{\prime} \beta} \underset{B\left(z, 2^{-m^{\prime}}\right)}{f} u \mathrm{~d} x \leqslant 2 \gamma \text { for all } m^{\prime}=m, m+1, \ldots\right\}, \\
& W_{m}^{j}=\left\{z \in \Omega:\left|\left\{u>\tau^{-j}\right\} \cap B\left(z, 2^{-m}\right)\right|>\frac{1}{2}\left|B\left(z, 2^{-m}\right)\right|\right\}, \\
& Z_{m}^{j}=Z_{m} \cap W_{m}^{j} .
\end{aligned}
$$

As in [7] we can find $k \in \mathbb{N}$ and a compact set $Z^{*} \subset Z_{k}$ such that

$$
\frac{\log (4 \gamma)}{\log \tau}<k \text { and } H^{n-p}\left(Z^{*}\right)>\mu .
$$

In view of (2.2) we can also suppose that

$$
\lambda_{2^{-k+1}}^{n-p}\left(Z^{*}\right)>\mu \quad \text { and } \quad \frac{\log (1 / \delta)}{\log \tau}<k
$$

(this can only increase the value of $k$ and therefore (3.6) remains valid). From now on this $k \in \mathbb{N}$ is fixed. Since $Z^{*}$ is compact and $Z^{*} \subset \bigcup_{j} W_{k}^{j}$, where $W_{k}^{1} \subset W_{k}^{2} \subset \cdots$ are open sets, we infer that there is $i \geqslant 2 k$ such that

$$
Z^{*} \subset W_{k}^{i} \text {. }
$$


We denote

$$
P_{k}^{j}=\bigcup_{m \geqslant k} Z_{m}^{j-1} \backslash Z_{m+1}^{j} .
$$

With each $z \in P_{k}^{j}$ we associate a ball $B_{z}=B\left(z, 2^{m}\right)$ where $m$ is such that $z \in A_{m}^{j}, m \geqslant k$. By the Besicovitch covering theorem we find a countable system $\mathcal{B}_{k}^{j} \subset\left\{B_{z}: z \in Z_{m}^{j-1} \backslash Z_{m+1}^{j}, m \geqslant k\right\}$ such that

$$
\chi_{P_{k}^{j}} \leqslant \sum_{B \in \mathcal{B}_{k}^{j}} \chi_{B} \leqslant N
$$

Using the definition of the sets $Z_{m}^{j}$ we can deduce with some work that

$$
\lambda_{2^{-k+1}}^{n-p}\left(Z^{*}\right) \leqslant \frac{1}{a} \sum_{a<j \leqslant 3 a} \sum_{B \in \mathcal{B}_{k}^{j}}(\operatorname{diam} B)^{n-p}
$$

for any $a \in \mathbb{N}, a \geqslant i$. In fact this was proved in [7] (two lines down from formula (18) there) only for $a=i$ but it is easy to see that everything works well also for $a \geqslant i$. From (3.7) we obtain

$$
\mu \leqslant \frac{1}{a} \sum_{a<j \leqslant 3 a} \sum_{B \in \mathcal{B}_{k}^{j}}(\operatorname{diam} B)^{n-p}
$$

Fix $j \in\{a+1, \ldots, 3 a\}$ and set

$$
\begin{aligned}
& \ell:=\tau^{-j+1}, \\
& E_{j}:=\bigcup_{B \in \mathcal{B}_{k}^{j}} B \cap\{u \leqslant \ell\} \cap\left\{u>\tau^{-1} \ell\right\} .
\end{aligned}
$$

Note that the sets $E_{j}$ are clearly pairwise disjoint. Let $B=B(z, r) \in \mathcal{B}_{k}^{j}$ and $v=\max \left\{\tau^{-1} \ell, \min \{u, \ell\}\right\}$. Since $z \in P_{k}^{j}$, there exists $m \geqslant k$ such that $z \in Z_{m}^{j-1} \backslash Z_{m+1}^{j}$. Therefore we can use the Poincaré inequality in a standard way (see [7] for details) to deduce that

$$
\ell^{p} \leqslant C r^{p-n} \int_{B}|\nabla v|^{p} \mathrm{~d} x=C r^{p-n} \int_{B \cap E_{j}}|\nabla u|^{p} \mathrm{~d} x
$$

where $C=C(n, p, \beta)$. Since, for every $x \in E_{j}$, we have $u(x) \sim \ell \sim \tau^{-j}$ and $a<j \leqslant 3 a$ this implies

$$
\frac{C}{a} \leqslant r^{p-n} \int_{B \cap E_{j}} \frac{|\nabla u|^{p}}{u^{p} \log 1 / u} \mathrm{~d} x .
$$

We multiply both sides of (3.11) by $r^{n-p} / \mu \sim(\operatorname{diam} B)^{n-p} / \mu$ and sum over $B \in \mathcal{B}_{k}^{j}$ and then sum over $j \in$ $\{a+1, \ldots, 3 a\}$. Then, with the aid of (3.10), we arrive at

$$
C \leqslant C \mu^{-1} \frac{1}{a} \sum_{j=a+1}^{3 a} \sum_{B \in \mathcal{B}_{k}^{j}}(\operatorname{diam} B)^{n-p} \leqslant C^{\prime} \mu^{-1} \sum_{j=a+1}^{3 a} \int_{E_{j}} \frac{|\nabla u|^{p}}{u^{p} \log 1 / u} \mathrm{~d} x
$$

with $C^{\prime}=C^{\prime}(n, p, \beta, \mu)$ (the constant $C^{\prime}$ involves also the constant $N$ from the Besicovitch covering theorem). It follows from the estimate $u \leqslant \tau^{-j+1}$ on $E_{j}, i \geqslant k$ and (3.7) that

$$
\{0<u<\delta\} \supset \bigcup_{m=i+1}^{\infty} E_{m} .
$$


Therefore, the estimate $\tau^{-j} \leqslant|u| \leqslant \tau^{-j+1}$ on $E_{j}$, (3.3)(i), (3.12) and (3.3)(ii) imply

$$
\begin{aligned}
\int_{0<u<\delta} \frac{|\nabla u|^{p}}{u^{p} \log 1 / u \Phi(\log \log 1 / u)} \mathrm{d} x & \geqslant \sum_{m=i+1}^{\infty} \int_{E_{m}} \frac{|\nabla u|^{p}}{u^{p} \log 1 / u \Phi(\log \log 1 / u)} \mathrm{d} x \\
& \geqslant \sum_{l=0}^{\infty} \sum_{j=3^{l} i+1}^{3^{l+1} i} \int_{E_{j}} \frac{|\nabla u|^{p}}{u^{p} \log 1 / u \Phi(\log \log 1 / u)} \mathrm{d} x \\
& \geqslant \sum_{l=0}^{\infty} \frac{C}{\Phi\left(\log \left(3^{l+1} i \log (\tau)\right)\right)} \sum_{j=3^{l} i+1}^{3^{l+1} i} \int_{E_{j}} \frac{|\nabla u|^{p}}{u^{p} \log 1 / u} \mathrm{~d} x \\
& \geqslant \sum_{l=0}^{\infty} \frac{C}{\Phi(C(l+1))}=\infty
\end{aligned}
$$

Theorem 3.2. Assume that $1 \leqslant p<\tilde{p} \leqslant n$. Let $\Omega \subset \mathbb{R}^{n}$ be a connected open set and let $u \in W^{1, \tilde{p}}(\Omega)$ be $\tilde{p}$-quasicontinuous. Suppose that $u>0$ a.e. and $H^{n-p}(\{u=0\})>0$. Suppose further that a function $\tilde{\Phi}$ satisfies (1.2) (i) and (ii). Then, for each $0<\delta<\mathrm{e}^{-e}$,

$$
\int_{0<u<\delta} \frac{|\nabla u|^{p}}{u^{p} \tilde{\Phi}(\log 1 / u)} \mathrm{d} x=\infty .
$$

Proof. By Proposition 2.1 there exists $\gamma \in(0, \infty)$ such that $H^{n-p}\left(Z_{\gamma}\right)>0$, where

$$
Z_{\gamma}=\left\{z \in \Omega: \limsup _{r \rightarrow 0} r^{-\beta} f_{B(z, r)}|f| \mathrm{d} x<\gamma\right\} .
$$

Recall that $\beta=1-p / \tilde{p}$.

Define

$$
\Phi(t)=\frac{\tilde{\Phi}(\exp t)}{\exp t} .
$$

From (1.2)(i) for $\tilde{\Phi}$ we obtain (3.3)(i), and clearly

$$
\int_{1}^{\infty} \frac{\mathrm{d} t}{\Phi(t)}=\int_{1}^{\infty} \frac{\exp t \mathrm{~d} t}{\tilde{\Phi}(\exp t)}=\int_{e}^{\infty} \frac{\mathrm{d} s}{\tilde{\Phi}(s)}=\infty .
$$

Now Lemma 3.1 yields

$$
\infty=\int_{0<u<\delta} \frac{|\nabla u|^{p}}{u^{p} \log 1 / u \Phi(\log \log 1 / u)} \mathrm{d} x=\int_{0<u<\delta} \frac{|\nabla u|^{p}}{u^{p} \tilde{\Phi}(\log 1 / u)} \mathrm{d} x .
$$

The following elementary example shows that our assumption (1.2)(ii) is essential in Theorem 3.2.

Example 3.3. Let $n, p \in \mathbb{N}, \Omega=\left(\frac{-1}{e p}, \frac{1}{e p}\right)^{n}$ and suppose that $1 \leqslant p<n$. Set $u(x)=\sqrt{x_{1}^{2}+\cdots+x_{p}^{2}}$. Clearly

$$
H^{n-p}(\{u=0\})=H^{n-p}\left(\{0\}^{p} \times\left(\frac{-1}{e p}, \frac{1}{e p}\right)^{n-p}\right)>0 .
$$


Suppose that

$$
\int_{1}^{\infty} \frac{\mathrm{d} s}{\tilde{\Phi}(s)}<\infty
$$

Then

$$
\int_{\Omega} \frac{|\nabla u|^{p}}{u^{p} \tilde{\Phi}(\log 1 / u)}=2^{n-p} \int_{(-1 / e p, 1 / e p)^{p}} \frac{\mathrm{d} x}{|x|^{p} \tilde{\Phi}(\log 1 /|x|)} \leqslant C \int_{0}^{1 / e} \frac{\mathrm{d} t}{t \tilde{\Phi}(\log 1 / t)}=C \int_{1}^{\infty} \frac{\mathrm{d} s}{\tilde{\Phi}(s)}<\infty .
$$

\section{Hausdorff measure of $f^{-1}(0)$}

We need the following elementary inequalities that can be viewed as variants of Young's inequality.

Lemma 4.1. Let $\Psi:[1, \infty) \rightarrow[1, \infty)$ be a differentiable concave function and set $\psi(t):=\Psi^{\prime}(t)$. Suppose that the function

$$
t \rightarrow t^{n-1} \psi(\log (e+t))
$$

is increasing. Then

$$
\frac{c^{n-1} \psi(\log (e+1 / a))}{a^{n-1} \Psi(\log (e+1 / a))} \leqslant \frac{c^{n} \psi(\log (e+1 / a))}{b a^{n} \Psi^{n /(n-1)}(\log (e+1 / a))}+b^{n-1} \psi(\log (e+b / c)) .
$$

for every $a>0, b>0$ and $c>0$.

Proof. If the first term on the right-hand side of (4.1) is greater or equal to the left-hand side then the inequality is obvious. Otherwise

$$
b \geqslant \frac{c}{a \Psi^{1 /(n-1)}(\log (e+1 / a))} .
$$

Since the function $t^{n-1} \psi(\log (e+t))$ is increasing, $\psi$ is non-increasing and $\Psi \geqslant 1$, this implies

$$
\begin{aligned}
b^{n-1} \psi(\log (e+b / c)) & \geqslant \frac{c^{n-1}}{a^{n-1} \Psi(\log (e+1 / a))} \psi\left(\log \left(e+\frac{1}{a \Psi^{1 /(n-1)}(\log (e+1 / a))}\right)\right) \\
& \geqslant \frac{c^{n-1}}{a^{n-1} \Psi(\log (e+1 / a))} \psi(\log (e+1 / a)) .
\end{aligned}
$$

Lemma 4.2. Suppose that $\Phi:[1, \infty) \rightarrow(0, \infty)$ satisfies $(1.2)$ for $n=2$. Then

$$
a \leqslant \frac{a^{2} \Phi(\log (e+a / c))}{b}+C \frac{b}{\Phi(\log (e+b / c))}
$$

for every $a \geqslant 0, b>0$ and $c>0$.

Proof. If the first term on the right-hand side of (4.2) is greater or equal to the left-hand side then the inequality is obvious. Otherwise

$$
b \geqslant a \Phi(\log (e+a / c)) .
$$


From (1.2) (i) and (iv) it is easy to see that $\Phi$ increases at most like a power function. Therefore $\Phi(\log (e+t)) \leqslant$ $C+C t$ for every $t>0$. With the help of (1.2)(iv) this implies

$$
\Phi(\log (e+t \Phi(\log (e+t)))) \leqslant C \Phi(\log (e+t)) .
$$

From (1.2)(iii), (4.3) and (4.4) we have

$$
\frac{b}{\Phi(\log (e+b / c))} \geqslant \frac{a \Phi(\log (e+a / c))}{\Phi(\log (e+a / c \Phi(\log (e+a / c))))} \geqslant C a .
$$

Proof of Theorem 1.2. Suppose that $f$ is not identically 0 and that $H^{1}\left(f^{-1}(0)\right)>0$. We know that there is $0<\delta<\mathrm{e}^{-e}$ such that the multiplicity of $f$ is bounded almost everywhere on $B(0, \delta)$ by constant $M>0$.

Set

$$
\Psi(t)=1+\int_{1}^{t} \frac{\mathrm{d} s}{\Phi(s)} \quad \text { and } \quad \psi(t)=\Psi^{\prime}(t)=\frac{1}{\Phi(t)} \quad \text { for } t \geqslant 1 .
$$

From (1.2)(ii) we know that $\lim _{t \rightarrow \infty} \Psi(t)=\infty$ and therefore also

$$
\infty=\lim _{t \rightarrow \infty} \log \Psi(t)=\lim _{t \rightarrow \infty} \int_{1}^{t} \frac{\psi(s) \mathrm{d} s}{\Psi(s)}=\int_{1}^{\infty} \frac{\mathrm{d} s}{\Phi(s) \Psi(s)} .
$$

Hence the function $\tilde{\Phi}(t)=\Phi(t) \Psi(t)$ satisfies assumptions (1.2) (i) and (ii). We wish to apply Theorem 3.2 to $|f|$ for $p=n-1$. In order to do this we still need to check that $\left|f^{-1}(0)\right|=0$. When $n=2$ this follows from Lemma 4.3 below and for $n \geqslant 3$ from formula (2.3) in [15]; notice that this result can be applied because (1.1) and (1.2) imply that $K^{1 /(n-1)} \in L_{\text {loc }}^{1}(\Omega)$. We thus obtain from Theorem 3.2, for $p=n-1$ and $u=|f|$, that

$$
\int_{0<|f|<\delta} \frac{|D f|^{n-1} \psi(\log (e+1 /|f|))}{|f|^{n-1} \Psi(\log (e+1 /|f|))}=\infty
$$

Denote $\Omega_{0}=\Omega \cap\{|D f(x)| \neq 0\} \cap\{0<|f|<\delta\}$. It is not difficult to verify from (4.5) and (1.2) that $\Psi$ satisfies all the assumptions of Lemma 4.1. We use inequality (4.1) for $a(x)=|f(x)|, b(x)=K(x)$ and $c(x)=|D f(x)|$ to obtain the estimate

$$
\begin{aligned}
& \int_{\Omega_{0}} \frac{|D f|^{n-1} \psi(\log (e+1 /|f|))}{|f|^{n-1} \Psi(\log (e+1 /|f|))} \\
& \quad \leqslant \int_{\Omega_{0}} \frac{|D f|^{n} \psi(\log (e+1 /|f|))}{K|f|^{n} \Psi^{n /(n-1)}(\log (e+1 /|f|))}+\int_{\Omega_{0}} K^{n-1} \psi(\log (e+K /|D f|)) .
\end{aligned}
$$

Since $\psi$ is non-increasing, part (iv) of (1.2) and our integrability assumptions give us

$$
\begin{aligned}
\int_{\Omega_{0}} K^{n-1} \psi(\log (e+K /|D f|)) & \leqslant \int_{K \leqslant|D f|^{s /(n-1)}}+\int_{K>|D f|^{s /(n-1)}} \\
& \leqslant \psi(1) \int_{\Omega}|D f|^{s}+\int_{\Omega} K^{n-1} \psi\left(\log \left(e+K^{(s-n+1) / s}\right)\right) \\
& \leqslant C \int_{\Omega}|D f|^{s}+C \int_{\Omega} K^{n-1} \psi(\log (e+K)) \leqslant C .
\end{aligned}
$$


Using the distortion inequality, (2.1) and $\lim _{s \rightarrow \infty} \Psi(s)=\infty$ we conclude that

$$
\begin{aligned}
& \int_{\Omega_{0}} \frac{|D f|^{n} \psi(\log (e+1 /|f|))}{K|f|^{n} \Psi^{n /(n-1)}(\log (e+1 /|f|))} \leqslant \int_{\Omega_{0}} \frac{J_{f}(x) \psi(\log (e+1 /|f|))}{|f|^{n} \Psi^{n /(n-1)}(\log (e+1 /|f|))} \\
& \quad \leqslant C M \int_{B(0, \delta)} \frac{\psi(\log (e+1 /|y|))}{|y|^{n} \Psi^{n /(n-1)}(\log (e+1 /|y|))} \mathrm{d} y=C \int_{0}^{\delta} \frac{\psi(\log (e+1 / t))}{t \Psi^{n /(n-1)}(\log (e+1 / t))} \mathrm{d} t \\
& \leqslant C\left(\Psi^{-1 /(n-1)}(\log (e+1 / \delta))-\lim _{t \rightarrow 0+} \Psi^{-1 /(n-1)}(\log (e+1 / t))\right) \leqslant C .
\end{aligned}
$$

Combining (4.7), (4.8) and (4.9) we arrive at

$$
\int_{0<|f|<\delta} \frac{|D f|^{n-1} \psi(\log (e+1 /|f|))}{|f|^{n-1} \Psi(\log (e+1 /|f|))}=\int_{\Omega_{0}} \frac{|D f|^{n-1} \psi(\log (e+1 /|f|))}{|f|^{n-1} \Psi(\log (e+1 /|f|))} \leqslant C .
$$

This clearly contradicts (4.6).

We close this section by verifying the following result that was employed in the proof above.

Lemma 4.3. Suppose that $n=2$ and that $f$ is as in Theorem 1.2. Then $\left|f^{-1}(0)\right|=0$.

Proof. By Lemma 5.1 in [11] we may find a (radial) function $u \in W_{0}^{1, n}(B(0,1))$ so that $\lim _{y \rightarrow 0} u(y)=\infty$ and

$$
\int_{B(0,1)}|\nabla u|^{2} \Phi(\log (e+|\nabla u|))<\infty
$$

Therefore we can find a decreasing sequence of numbers $R_{k} \searrow 0$ and sequence of functions $u_{k} \in W_{0}^{1, n}\left(B\left(0, R_{k}\right)\right)$ so that $u_{k} \equiv 1$ on $B\left(0, R_{k+1}\right)$ and

$$
\lim _{k \rightarrow \infty} \int_{B\left(0, R_{k}\right)}\left|\nabla u_{k}\right|^{2} \Phi\left(\log \left(e+\left|\nabla u_{k}\right|\right)\right)=0 .
$$

Since $f$ is quasi-light, we may assume that $\Omega$ is bounded, $f^{-1}(0)$ is compact and there exists $\delta>0$ such that $f^{-1}(B(0, \delta)) \Subset \Omega$ (cf. [25, Theorem 3.1]).

For $k \in \mathbb{N}$ we write

$$
B_{k}=B\left(0, R_{k}\right), \quad A_{k}=f^{-1}\left(B_{k} \backslash B_{k+1}\right) \quad \text { and } \quad \tilde{A}_{k}=A_{k} \cap\{|D f| \neq 0\} .
$$

Fix $k \in \mathbb{N}$ large and denote $v=u_{k} \circ f$. If $R_{k}<\delta$, then the function $v$ has zero boundary values and thus we may use the Sobolev inequality for $v$. Since $u_{k}(0) \equiv 1$ on $B_{k+1}$ we obtain from the Sobolev inequality and (4.2) for $a=|\nabla v|, b=K$ and $c=|D f|$ that

$$
\begin{aligned}
\left|f^{-1}(0)\right| & \leqslant \int_{\Omega}\left|u_{k} \circ f\right| \leqslant C \int_{\Omega}|\nabla v|=\int_{\tilde{A}_{k}}|\nabla v| \\
& \leqslant \int_{\tilde{A}_{k}} \frac{|\nabla v|^{2}}{K} \Phi(\log (e+|\nabla v| /|D f|))+C \int_{\tilde{A}_{k}} \frac{K}{\Phi(\log (e+K /|D f|))} .
\end{aligned}
$$


Analogously to (4.8) we obtain

$$
\int_{\tilde{A}_{k}} \frac{K}{\Phi(\log (e+K /|D f|))} \leqslant C \int_{\tilde{A}_{k}}|D f|^{s}+C \int_{\tilde{A}_{k}} \frac{K}{\Phi(\log (e+K))} .
$$

The right-hand side of (4.12) tends to zero when $k \rightarrow \infty$ because the sets $A_{k}$ are clearly pairwise disjoint. For $k$ large enough, the multiplicity of $f$ is essentially bounded by $M$ on $B_{k}$. Therefore we can use the distortion inequality, (2.1) and (4.10) to obtain

$$
\begin{aligned}
\int_{\tilde{A}_{k}} \frac{|\nabla v|^{2}}{K} \Phi(\log (e+|\nabla v| /|D f|)) & \leqslant \int_{\tilde{A}_{k}}\left|\left(\nabla u_{k}\right) \circ f\right|^{2} J_{f} \Phi\left(\log \left(e+\left|\left(\nabla u_{k}\right) \circ f\right|\right)\right) \\
& \leqslant M \int_{B_{k}}\left|\nabla u_{k}\right|^{2} \Phi\left(\log \left(e+\left|\nabla u_{k}\right|\right)\right) \underset{k \rightarrow \infty}{\rightarrow} 0 .
\end{aligned}
$$

From (4.11), (4.12) and (4.13) we obtain $\left|f^{-1}(0)\right|=0$.

\section{Openness and discreteness}

In this section we prove discreteness and openness of a mapping under a weaker integrability condition on $D f$ than $D f \in L^{n}$. We use an Orlicz-type condition that was introduced to this setting in [16,8], and [14].

Theorem 5.1. Let $\Omega \subset \mathbb{R}^{n}$ be a connected open set and suppose that $n-1<p \leqslant n$. Let $f \in W_{\mathrm{loc}}^{1, p}\left(\Omega ; \mathbb{R}^{n}\right)$ be a continuous, sense-preserving mapping that has essentially bounded multiplicity. Suppose that $f$ is a mapping of finite distortion $K$ so that $K$ satisfies (1.1) with a function $\Phi$ that satisfies (1.2). Then $f$ is either constant or both discrete and open.

Proof. Any sense-preserving, light and continuous mapping is both discrete and open, see [24] or [23, Lemma 5.6]. Hence it remains to show that $f$ is light. However, by Theorem $1.2, H^{1}\left(f^{-1}(y)\right)=0$ for each $y \in \mathbb{R}^{n}$, which easily implies lightness.

Now let us state the main result of our paper. Theorem 1.1 follows from Theorem 5.2 by choosing $\Psi(t)=t^{n}$.

Theorem 5.2. Let $\Omega \subset \mathbb{R}^{n}$ be a connected open set. Let $\Psi$ be a non-negative, strictly increasing and continuously differentiable function on $[0, \infty)$ satisfying the conditions

$$
\int_{1}^{\infty} \frac{\Psi(t)}{t^{1+n}} \mathrm{~d} t=\infty, \quad \liminf _{t \rightarrow \infty} \frac{t \Psi^{\prime}(t)}{\Psi(t)}>s
$$

with $s>n-1$. Suppose that $f$ is a quasi-light mapping of finite distortion $K$ that satisfies (1.1) with a function $\Phi$ that satisfies (1.2), and suppose further that $\Psi(D f) \in L_{\mathrm{loc}}^{1}(\Omega)$. Then $f$ is discrete and open.

Proof. Using results from [9] (or [5,16]) and [12] we may obtain analogously to [7, Theorem 5 and 7] that $f$ is sense-preserving, and that each point $x_{0} \in \Omega$ is contained in a subdomain $\Omega^{\prime \prime} \subset \Omega$ such that $N\left(f, \Omega^{\prime \prime}, \cdot\right)$ is essentially bounded. Thus we may use Theorem 5.1 to show that $f$ is open and discrete on $\Omega^{\prime \prime}$. Since these properties are local, the proof is complete. 
Let us close the paper by commenting on the sharpness of our assumptions. As discussed in the introduction, our assumption on $K$ is optimal. Moreover, an example constructed in [14] gives us, given $\Psi$ with

$$
\int_{1}^{\infty} \frac{\Psi(t)}{t^{1+n}} \mathrm{~d} t<\infty,
$$

a non-discrete, non-open, quasi-light mapping of finite distortion $K$ so that $K \in L^{p}(\Omega)$ for all $p$, in particular for $p=n-1$, and so that $\Psi(D f)$ is in $L_{\text {loc }}^{1}(\Omega)$. Thus the integrability assumption on $|D f|$ is also optimal.

\section{References}

[1] J. Ball, Global invertibility of Sobolev functions and the interpenetration of matter, Proc. Roy. Soc. Edinburgh Sect. A 88 (3-4) (1981) $315-328$.

[2] H. Federer, Geometric Measure Theory, Grundlehren Math. Wiss., vol. 153, Springer-Verlag, New York, 1969 (second edition, 1996).

[3] I. Fonseca, W. Gangbo, Degree Theory in Analysis and Applications, Clarendon Press, Oxford, 1995.

[4] V. Gol'dstein, S. Vodop'yanov, Quasiconformal mappings and spaces of functions with generalized first derivatives, Sibirsk. Mat. Zh. 17 (1976) 515-531.

[5] L. Greco, Sharp integrability of nonnegative Jacobians, Rend. Mat. 18 (1998) 585-600.

[6] J. Heinonen, P. Koskela, Sobolev mappings with integrable dilatations, Arch. Rational Mech. Anal. 125 (1) (1993) 81-97.

[7] S. Hencl, J. Malý, Mappings of finite distortion: Hausdorff measure of zero sets, Math. Ann. 324 (2002) 451-464.

[8] T. Iwaniec, P. Koskela, J. Onninen, Mappings of finite distortion: monotonicity and continuity, Invent. Math. 144 (2001) $507-531$.

[9] T. Iwaniec, G. Martin, Geometric Function Theory and Nonlinear Analysis, Oxford Mathematical Monographs, Clarendon Press, Oxford, 2001.

[10] T. Iwaniec, V. Šverák, On mappings with integrable dilatation, Proc. Amer. Math. Soc. 118 (1993) 181-188.

[11] J. Kauhanen, P. Koskela, J. Malý, On functions with derivatives in a Lorentz space, Manuscripta Math. 100 (1999) 87-101.

[12] J. Kauhanen, P. Koskela, J. Malý, Mappings of finite distortion: discreteness and openness, Arch. Ration. Mech. Anal. 160 (2001) $135-151$.

[13] J. Kauhanen, P. Koskela, J. Malý, Mappings of finite distortion: condition N, Michigan Math. J. 49 (2001) 169-181.

[14] J. Kauhanen, P. Koskela, J. Malý, J. Onninen, X. Zhong, Mappings of finite distortion: sharp Orlicz-conditions, Rev. Mat. Iberoamericana 19 (2003) 857-872.

[15] P. Koskela, J. Malý, Mappings of finite distortion: the zero set of the Jacobian, J. Eur. Math. Soc. (JEMS) 5 (2003) 95-105.

[16] P. Koskela, X. Zhong, Minimal assumptions for the integrability of the Jacobian, Ricerche Mat. L I (2002) 297-311.

[17] J. Malý, O. Martio, Lusin's condition (N) and mappings of the class $W^{1, n}$, J. Reine Angew. Math. 458 (1995) $19-36$.

[18] J. Malý, D. Swanson, W.P. Ziemer, Coarea formula for Sobolev mappings, Trans. Amer. Math. Soc. 355 (2) (2003) $477-492$.

[19] J. Malý, W.P. Ziemer, Fine Regularity of Solutions of Elliptic Partial Differential Equations, Amer. Math. Soc., Providence, RI, 1997.

[20] J. Manfredi, E. Villamor, An extension of Reshetnyak's theorem, Indiana Univ. Math. J. 47 (3) (1998) 1131-1145.

[21] Yu.G. Reshetnyak, Space mappings with bounded distortion, Sibirsk. Mat. Zh. 8 (1967) 629-658.

[22] Yu.G. Reshetnyak, Space Mappings with Bounded Distortion, Transl. Math. Monographs, vol. 73, Amer. Math. Soc., 1989.

[23] S. Rickman, Quasiregular Mappings, Ergeb. Math. Grenzgeb. (3) (Results in Mathematics and Related Areas (3)), vol. 26, Springer-Verlag, Berlin, 1993.

[24] C. Titus, G. Young, The extension of interiority, with some applications, Trans. Amer. Math. Soc. 103 (1962) 329-340.

[25] J. Väisälä, Minimal mappings in Euclidean spaces, Ann. Acad. Sci. Fenn. Ser. A I 366 (1965) 1-22.

[26] M. Vuorinen, Conformal Geometry and Quasiregular Mappings, Lecture Notes in Math., vol. 1319, Springer-Verlag, Berlin, 1988. 